\title{
Neutron stars accreting the ISM: Are they fast or slow objects ?
}

\author{
A. Treves ${ }^{\mathrm{a}}$, M. Colpi ${ }^{\mathrm{b}}$, R. Turollac ${ }^{\mathrm{c}}$ and S. Zane ${ }^{\mathrm{d}}$ \\ aII Faculty of Sciences, University of Milano Via Lucini 3, 22100 Como, Italy \\ ${ }^{\mathrm{b}}$ Dept. of Physics, University of Milano, Via Celoria 16, 20133 Milano, Italy \\ ${ }^{\mathrm{c}}$ Dept. of Physics, University of Padova, Via Marzolo 8, 35131 Padova, Italy \\ dNuclear and Astrophysics Laboratory, University of Oxford, Keble Road, Oxford OX1 3RH, UK
}

\begin{abstract}
Old neutron stars (ONSs) which have radiated away their internal and rotational energy may still shine if accreting the interstellar medium. Rather stringent limits from the analysis of ROSAT surveys indicate that most optimistic predictions on ONSs observability are in excess of a factor as large as $\sim 100$. Here we explore two possible evolutionary scenarios that may account for the paucity of ONSs. In the first it is assumed that the ONS population is not too fast $\left(V<100 \mathrm{~km} \mathrm{~s}^{-1}\right)$ and that magnetic field decay guides the evolution. In the second, NSs move with high speed $\left(V>100 \mathrm{~km} \mathrm{~s}^{-1}\right)$ and preserve their magnetic field at birth. We find that according to the former scenario most ONSs are now in the propeller phase, while in the latter nearly all ONSs are silent, dead pulsars.
\end{abstract}

\section{ONSs Basics}

Isolated old neutron stars (ONSs), i.e. stars which have overcome the pulsar phase, are expected to be as many as $N \sim 10^{9}$ in the Galaxy. Ostriker, Rees, \& Silk [1] were the first to suggest that accretion of the interstellar medium (ISM) may produce enough luminosity to make the closest stars observable. Assuming that accretion proceeds at the Bondi rate

$\dot{M} \simeq 10^{11} n v_{10}^{-3} \mathrm{~g} \mathrm{~s}^{-1}$,

where $v_{10}=\left(V_{10}^{2}+c_{s, 10}^{2}\right)^{1 / 2}, V_{10}$ is the ONS velocity in units of $10 \mathrm{~km} \mathrm{~s}^{-1}, c_{s} \sim 10 \mathrm{kms}^{-1}$ is the ISM sound speed and $n$ the ISM number density in $\mathrm{cm}^{-3}$, the total luminosity is

$L \simeq 7 \times 10^{31} n v_{10}^{-3} \mathrm{erg} \mathrm{s}^{-1}$.

In the case of blackbody emission from the polar caps, the emitted radiation spectrum peaks around

$T_{\text {eff }} \simeq 10^{6} L_{31} B_{9}^{1 / 7} n^{-1 / 14} v_{10}^{3 / 14} \mathrm{~K}$

where $B_{9}$ is the surface magnetic field in units of $10^{9} \mathrm{G}$.

Treves, \& Colpi [2] and Blaes, \& Madau [3] estimated that, under rather favorable conditions, several thousands ONSs should appear in the ROSAT All Sky Survey and a few in each deep field. However, despite the intense observational efforts, the search for ONSs produced, up to now, just a handful of candidates, out of which only two, RX J18653.5-3754 [1] and RX J0720.4-3125 [5.6] seem promising. Moreover, recent analyses of ROSAT fields in the direction of Giant Molecular Clouds [7,8] placed rather stringent upper limits on the number of ONSs in ROSAT images indicating that current theoretical predictions are in excess of at least a factor $\sim 5-10$. A even more dramatic reduction of the number of ONSs could follow from the recent investigation of ROSAT sources in dark clouds and high latitude molecular clouds by Danner [9]. For these fields predictions seem to be in excess by a factor $\sim 10-100$.

\section{Why Are ONSs Elusive?}

Several facts may actually conspire to make detectable ONSs rare.

a) ONSs could be a faster population than what assumed in previous studies which relied on the Narayan \& Ostriker 10 velocity distribution at birth, because either the pulsar distribution peaks 
at higher velocities 11] or of successive dynamical heating [12]. The higher the average speed, the lower the luminosity, since it is $L \propto v^{-3}$. Very recent studies 13, 14 seem to confirm that far less pulsars populate the low-velocity tail $\left(V<100 \mathrm{~km} \mathrm{~s}^{-1}\right)$, relative to Narayan \& Ostriker's distribution (see however 15]).

b) The X-ray radiation produced in the accretion process may heat the surrounding medium [16]. After an initial "burst", the sound speed in the ISM becomes large enough to cut drastically the accretion rate (see eq. [1]).

c) The assumptions that ONSs have only a relic field $\sim 10^{9} \mathrm{G}$ and that all of them are in the accretion stage now oversimplify the picture. The number of ONSs accreting the ISM may depend sensitively on the spin and magnetic field evolution.

\section{Slowly moving ONSs with decaying magnetic field}

Treves, Colpi \& Lipunov 17 and Blaes \& Madau 3 noted that for accretion to be possible, the NS must have spun down significantly. Spin-down is provided first by dipole losses in the pulsar phase and subsequently by the interaction of the rotating magnetosphere with the incoming material in the propeller phase (see [18]). Only when the rotational velocity is low enough to make the centrifugal pull no longer effective, accretion onto the star surface can occur. Since the spin-down rates depend on both the star magnetic field and the period, the "ability" to accrete is strongly related to the evolution of $B$ and $P$. Livio, Xu \& Frank [19 and Colpi, Turolla, Zane \& Treves 20 (CTZT hereafter) proposed that the deficiency of detected ONSs is related to the magnetic field and its decay, and is explained in terms of a global reduction in the number of accreting objects. They have shown that magnetic field decay reduces the efficiency of spin-down (both by dipole radiation and by propeller) to such an extent that the star never enters the accretion stage. Under rather general assumptions, and regardless of the details of field evolution (ohmic decay of a crustal field or spin-induced decay of the core field), the number of accretors decreases by more than one order of magnitude if the characteristic decay time is $\approx 10^{8} \mathrm{yr}$. In addition a large fraction of ONSs would be in the propeller state. These models are successful in explaining the spin period $(P=8.39 \mathrm{~s})$ observed in the ONS candidate RXJ0720.4-3125 [6,21,22]. CTZT also noticed that nearly all ONSs would be accreting today, if some physical processes in the crust and/or in the NS interior prevent $B$ from decaying substantially, and this would contrast with existing observational limits.

\section{Fastly moving ONSs with non-decaying magnetic field}

Because of the uncertainties in the velocity distribution of pulsars, and hence of ONSs, it may be useful to discuss the status of ONSs observability considering a distribution function devoided of low-velocity stars. In the following we adopt a different view for the evolution of the $B$-field, assuming that ONSs retain their magnetic field at birth. Since no decay on timescales shorter than the typical pulsar lifetime $\left(\approx 10^{7}\right.$ yr $)$ has been observed so far and because decay models are far from giving univocal predictions, the nodecay scenario is at least as plausible as the opposite one.

Here we consider the evolution of a NS with mass $M=1 M_{\odot}, R=10 \mathrm{~km}$ and moment of inertia $I=0.8 \times 10^{45} \mathrm{~g} \mathrm{~cm}^{2} . B_{12}$ is the constant field in units of $10^{12} \mathrm{G}, P$ is the star rotational period and the notation of CTZT is used. Over its lifetime, a NS goes through three main evolutionary phases which are discussed in some detail below.

\subsection{Emitter phase}

This regime begins with the pulsar phase and proceeds also after the break-down of the coherence condition which quenches the radio emission (the star becomes a dead, or silent, pulsar). The energy losses are due to magnetic dipole radiation and the period increases in time according to

$P=3 \times 10^{-4} B_{12} t^{1 / 2} \mathrm{~s}$

where $t$ is in yr. 


\subsection{Propeller phase}

When the gravitational energy density of the incoming interstellar gas exceeds the outward momentum flux of the star at the accretion radius, $r_{a c} \simeq G M / v^{2}$, matter starts to accrete. For this condition to be met, the period must have reached a critical value

$P_{\text {prop }} \simeq 10 n^{-1 / 4} v_{10}^{1 / 2} B_{12}^{1 / 2} \mathrm{~s}$

which is attained by dipole braking in a time

$t_{e} \simeq 1.1 \times 10^{9} n^{-1 / 2} v_{10} B_{12}^{-1} \mathrm{yr}$.

When $P>P_{\text {prop }}$ matter can penetrate down to the Alfven radius, $r_{A} \simeq 1.7 \times$ $10^{10} n^{-2 / 7} v_{10}^{6 / 7} B_{12}^{4 / 7} \mathrm{~cm}$, but the interaction with the rotating magnetosphere prevents accretion to go any further because of the centrifugal barrier. The NS is now in the propeller phase, rotational energy is lost to the ISM and the period keeps increasing at a rate

$\frac{d P}{d t} \simeq K P^{\alpha} \mathrm{s} \mathrm{yr}^{-1}$

with $K=\times 10^{-8} n^{9 / 13} v_{10}^{-27 / 13} B_{12}^{8 / 13}$ and $\alpha=$ $21 / 13$.

\subsection{Accretor phase}

Accretion to the star surface occurs when the corotation radius $r_{c o}=\left(G M P^{2} / 4 \pi^{2}\right)^{1 / 3}$ becomes larger than the Alfven radius. This implies that spin-down increased the period up to

$P_{a c} \simeq 2.5 \times 10^{3} n^{-3 / 4} v_{10}^{1 / 7} B_{12}^{6 / 7} \mathrm{~s}$.

Using equations (7) and (8), the duration of the propeller phase turns out to be

$t_{p}=\frac{P_{p r o p}^{1-\alpha}-P_{a c}^{1-\alpha}}{K(\alpha-1)} \mathrm{yr}$.

The two relevant timescales $t_{e}$ and $t_{p}$, the duration of the emitter and the propeller phase, depend on the star velocity, the ISM density and the magnetic field. In order for the star to become an accretor, a time $t_{a}=t_{e}+t_{p}$ has to elapse. Imposing the condition that each of the times is less (or equal) to the star lifetime, which we assume to coincide with the age of the galaxy $t_{G}=10^{10} \mathrm{yr}$, gives the velocity range within which the NS is, at present, a dead pulsar, a propellor or an accretor. Actually the condition that $P \geq P_{a c}$ [eq.(8)] is not sufficient to guarantee that matter is captured at the accretion radius. At the very low accretion rates expected for fast, isolated ONSs, it could be that the Alfven radius is larger that the accretion radius. The condition $r_{A}<r_{a c}$ translates into a limit for the star velocity

$v_{10}<25 n^{1 / 10} B_{12}^{-1 / 5}$

which needs to be taken into account together with $t_{p}<t_{G}$ and $t_{a}<t_{G}$. Results are shown in figures 11 and 2 where the velocities at which $t_{e}=t_{G}$ (solid line) and $t_{a}=t_{G}$ (dashed line), together with the limit implied by (10) (dashdotted line), are plotted against the star magnetic field for $n=1 \mathrm{~cm}^{-3}$ and $n=0.1 \mathrm{~cm}^{-3}$.

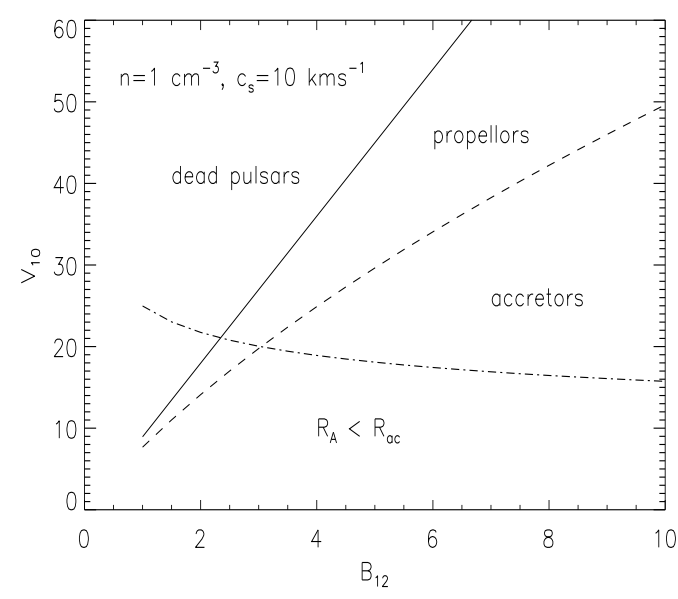

Figure 1. The different phases of ONSs at present as a function of the star velocity and magnetic field.

\section{Discussion}

As can be seen from figure 1, low-velocity ONSs $\left(V<70 \mathrm{~km} \mathrm{~s}^{-1}\right)$ moving through the average ISM are in the accretion phase now for all 


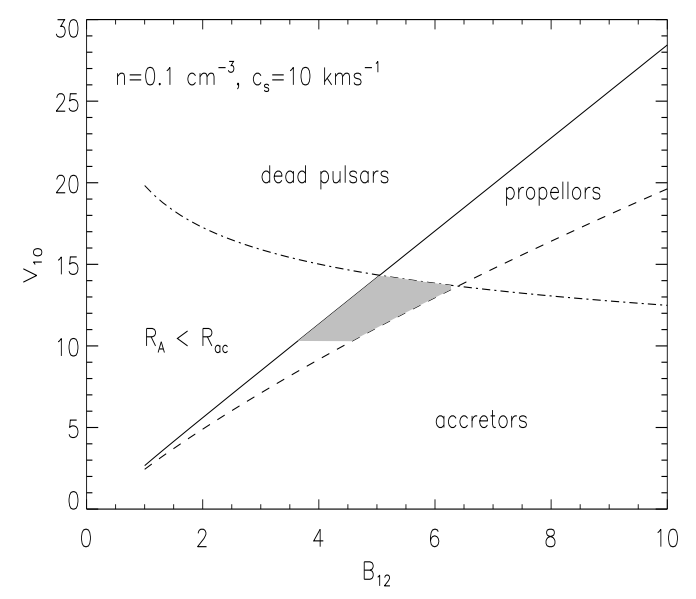

Figure 2. Same as in figure 1 for $n=0.1 \mathrm{~cm}^{-3}$.

reasonable values of the (constant) magnetic field. If their velocity distribution at birth is like that of Narayan \& Ostriker, there is an excess of accretors with respect to what implied by ROSAT observations. As discussed in $\S 3$, a possible way out is to invoke some form of field decay on timescales $<10^{9} \mathrm{yr}$. In this case, CTZT have found that for spin-induced decay the number of propellers may exceed that of accretors.

On the other hand, if typical ONSs velocities are $>100 \mathrm{~km} \mathrm{~s}^{-1}$, as favoured by most recent observations of pulsars velocities, most of them should have moved at relatively high Galactic latitudes, $z \sim 300 \mathrm{pc}$ or larger. In this case an appropriate value of the ISM density should be $0.1 \mathrm{~cm}^{-3}$ (see figure 2). All ONSs with $V>$ $150-200 \mathrm{~km} \mathrm{~s}^{-1}$ are above the limit $r_{A}=r_{a c}$ and will never be detectable, being unable to accrete. Although also fast stars with large enough fields $\left(B_{12}>5\right)$ may accrete, their luminosity will be much less than that of slow accretors, and the expectation of observing one of these objects is practically nil. There is, however, the possibility that the objects which populate the propeller region (the shaded area in figure 2) may be detectable. Even if their number is not expected to be large, the duration of the propeller phase is typically about $10^{9} \mathrm{yr}$, so there could be the possibility of catching these sources active. In fact, as proposed by Treves, Colpi \& Lipunov [17], ONSpropellers may exhibit a flaring activity due to episodic accretion of the matter which piles up at the Alfven radius. The flaring luminosity can be high, so one can hope to detect these transient sources.

\section{REFERENCES}

1. Ostriker, J.P., Rees, M.J., \& Silk, J. 1970, Astrophys. Letters, 6, 179

2. Treves, A., \& Colpi, M. 1991, A\&A, 241, 107

3. Blaes, O., \& Madau, P. 1993, ApJ, 403, 690

4. Walter, F.M., Wolk, S.J., \& Neuhäuser, R. 1996, Nature, 379, 233

5. Haberl, F., Pietsch, W., Motch, C., \& Buckley, D.A.H. 1996, IAUC No. 6445

6. Haberl, F., Motch, C., Buckley, D.A.H., Zickgraf, F.-J., \& Pietsch, W. 1997, A\&A, in press

7. Belloni, T., Zampieri, L., \& Campana, S. 1997, A\&A, 319, 525

8. Motch, C., et al. 1997, A\&A, 318, 111

9. Danner, R. 1996, unpublished PhD Thesis, Max-Planck Institut Für Extraterrestrische Physik, München

10. Narayan, R., \& Ostriker, J.P. 1990, ApJ, 270, 119

11. Lyne, A.G., \& Lorimer, D.R. 1994, Nature, 369,127

12. Madau, P., \& Blaes, O. 1994, ApJ, 423, 748

13. Cordes, J., \& Chernoff, D. 1997, ApJ, in press

14. Hansen, B.M.S., \& Phinney, E.S. 1997, MNRAS, submitted

15. Hartman, J.W. 1997, A\&A, 322,127

16. Blaes, O., Warren, R., \& Madau, P. 1995, ApJ, 454, 370

17. Treves, A., Colpi, M., \& Lipunov, V.M. 1993, A\&A, 269, 319

18. Illarionov, A., \& Sunyaev, R. 1975, A\&A, 39, 185

19. Livio, M., Xu, C., \& Frank, J. 1997, ApJ, in press

20. Colpi, M., Turolla, R., Zane, S., \& Treves, A. 1997, ApJ, submitted

21. Wang, J.C.L. 1997, ApJ, 486, L119 
22. Konenkov, D.Yu., \& Popov, S.B. 1997, preprint astro-ph/9707318 